\title{
Lift System Design of Tail-Sitter Unmanned Aerial Vehicle
}

\author{
Dizhou Zhang, Zili Chen, Junwei Lv \\ Optics and Electronic Department Mechanical Engineering College, Shijiazhuang, China \\ Email: oec_ljw2009@sohu.com
}

Received June 20, 2012; revised August 28, 2012; accepted September 4, 2012

\begin{abstract}
The main advantage of tail-sitter unmanned aerial vehicle (UAV) are introduced. Three design solutions of rotor tailsitter lift system of UAV have been presented and the respective control strategies and characteristics of three solutions are also analyzed in the paper, through the related experiments the design of twin-rotor lift system is verified, and its feasibility is proved. The characteristics and the applying background of the twin-rotor tail-sitter UAV are described in detail. Some useful conclusions of the lift system for tail-sitter UAV are obtained.
\end{abstract}

Keywords: Tail-Sitter UAV; Vertical Take-Off and Landing (VTOL); Rotor; Flight Control

\section{Introduction}

Quadrotor helicopter is a kind of vertical take-off and landing (VTOL) multi-rotor unmanned aerial vehicles (UAV). This kind of helicopter has many characteristics e.g., stable hovering and maneuverable flight in tough environment, its important advantage is load capacity. As these advantages, quadrotor helicopter has many utilities, such as search and rescue, building exploration, security and inspection, etc. especially in dangerous and inaccessible environments. So the aircraft has been widely used in various fields due to the advantages, such as air transport, crop monitoring and military reconnaissance. Conventional fixed-wing aircraft can fly at very high flight speed, but must rely on the runway in taking off and landing process. On the contrary, the helicopter can take off vertically out of the shackles of the runway, but its flight speed has been greatly hindered. After the Second World War, some companies in the United States, Canada and Europe started to develop a kind of vertical takeoff and landing (VTOL) aircraft which has the advantages of both fixed-wing aircraft and helicopter, obtaining high speed at the same time getting rid of the dependence on the runway.

VTOL aircraft can take off and land with zero velocity and hover in the air like a helicopter, and fly at high speed horizontally like fixed-wing aircraft [1]. The tailsitter UAV is a kind of superior performance VTOL aircraft due to its flexible handling and does not require complex thrust reversing mechanism.

The paper is organized as follows, Section 2 introduce the main advantage of tail-sitter UAV. Three kinds of lift system for tail-sitter UAV are presented and discussed in Section 3; Section 4 describes the twin-rotor tail-sitter
UAV in detail; Section 5 concludes the paper.

\section{Situation of VTOL Aircraft Research}

Compared with manned aircraft, the size and required power of the VTOL aircraft are relatively small, and the flight speed is low. Therefore, the power system of the VTOL aircraft mainly applies low-power turbine shaft and piston engine. Power system determines most of the VTOL UAV adopts the rotor. According to the implementation of the power system, the current rotor VTOL aircraft can be divided into two sorts, directional thrust aircraft and convertible thrust aircraft. The typical representative of the directional thrust aircraft is helicopter. The types of convertible thrust aircraft are tail-sitter, tilt-rotor, tilt-body, tilt-ducted fan, tilt-wing, etc.

Tail-sitter UAV vertically takes off to a certain height and turn into the horizontal flight mode. When it lands, it climbs with the nose up, and then reduces the thrust and lands vertically [2]. There are many unmanned aerial vehicles which apply this kind lift system, e.g., Golden Eye as shown in Figure 1. The thrust direction of tailsitter VTOL UAV is fixed to the vertical direction of airframe. When thrust converting, the direction of thrust and airframe turn over synchronously so that thrust convertible mechanism is realized simultaneously. The aircraft is light and simple, in the vertical flight condition; the quality of the airframe is mainly distributed in the vertical direction. The applied point of lift works on the barycenter, so the aircraft is natural stable. In the horizontal flight, it is easy to operate the UAV in conventional fixed-wing aircraft flight mode. In transition maneuver, the direction of the trust turns over synchronously with the airframe; and the converting process is 
simplified into the maneuver of the fixed-wing aircraft.

\section{Lift System Program of Tail-Sitter UAV}

The majority of the Tail-Sitter UAV relies on lift generated by rotors for flight. The lift system involves single rotor and two rotors. Changes of the lift in the size and direction will have a direct impact on the flight of the UAV, so the choice of lift system is very important for aircraft [3]. Here are three system layout programs of tail-sitter UAV's lift and the control methods of each program in vertical mode, transition maneuver and horizontal flight.

\subsection{Single-Rotor Tail-Sitter UAV Lift System}

In the single-rotor lift system, the rotor of the UAV is driven by an electrical motor. It balances the anti-torque of the rotor through the torque generating by four control surfaces which are submerged in the downwash of the propeller. The sketch map and the experimental model are as shown in Figure 2. During the flight, the UAV control the yaw motion, roll motion and pitch motion through the deflection of the control surface. By adjusting the rotor's speed and changing the power of the lift, UAV fly up and down and hover in the air. The control scheme is as shown in Table 1. Its lift system is a brush-

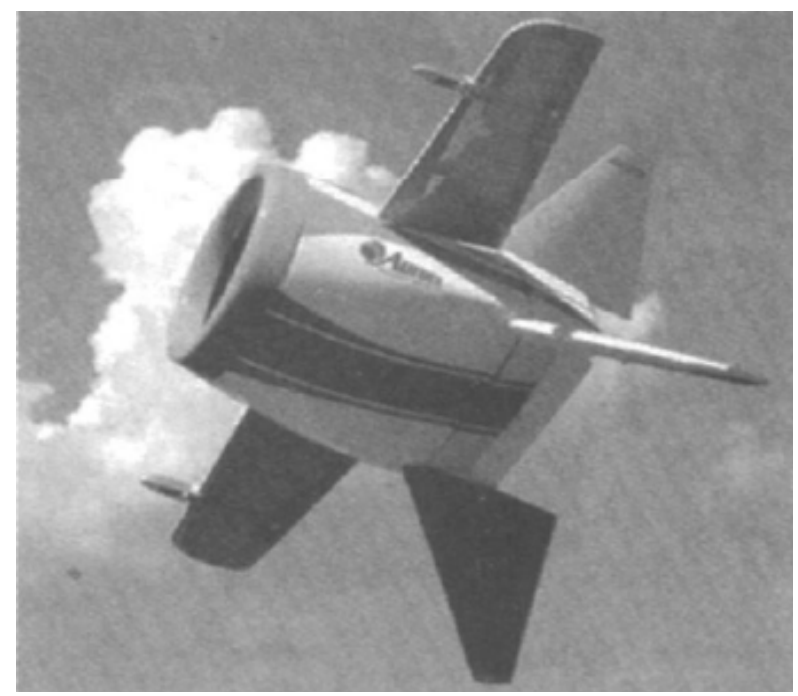

Figure 1. Golden eye UAV. less motor which is fixed to the top of the airframe. Four control surfaces are installed on four wings, submerged in the downwash of the propeller. In vertical flight and hover state, yaw motion is controlled relying on the clockwise or counterclockwise deflection of the four control surfaces. Pitch motion relies on the parallel deflection of the two control surfaces in the $\mathrm{Y}$ axis. Roll motion relies on the parallel deflection of the two control surfaces in the $\mathrm{X}$ axis. For achieving the transition maneuver from hover to horizontal flight, the deflection of the control surfaces in the $\mathrm{Y}$ axis is regulated by the control law until the vehicle reaches the desired pitch angle. In horizontal flight mode, the thrust of the aircraft is greatly reduced, and flight control is similar to the fixed-wing mode. Air moving over the outer body lift surface provides the lift for horizontal flight. Yaw motion is controlled by the parallel deflection of the two control surfaces in the $\mathrm{X}$ axis. Pitch motion relies on the parallel deflection of the two control surfaces in the $\mathrm{Y}$ axis; roll motion relies on the clockwise or counterclockwise deflection of four control surfaces.
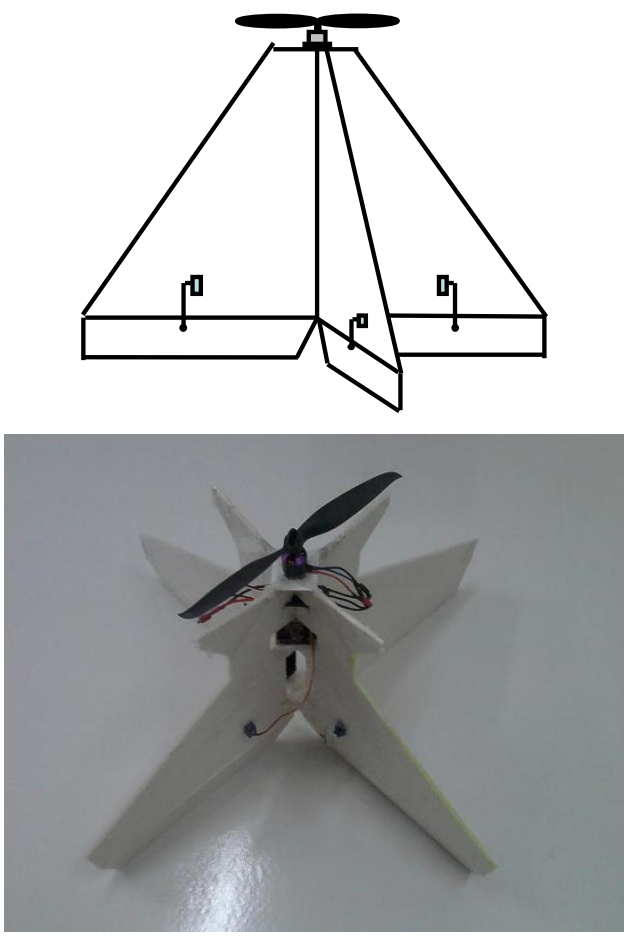

Figure 2. Single-rotor tail-sitter UAV.

Table 1. Control scheme of single-rotor tail-sitter UAV.

\begin{tabular}{ccc}
\hline & \multicolumn{1}{c}{ Vertical Mode } & Horizontal Mode \\
\hline Yaw Motio & Clockwiseor counterclockwise deflection of four control surfaces & Parallel deflection of the two control surfaces in the X axis \\
$\begin{array}{c}\text { Pitch Motion } \\
\text { Roll Motion }\end{array}$ & Parallel deflection of the two control surfaces in the Y axis & Parallel deflection of the two control surfaces in the Y axis \\
VTOL & Parallel deflection of the two control surfaces in the X axis & Clockwise or counterclockwise deflection of four control surfaces \\
\hline
\end{tabular}


In the single-rotor lift system, the control surfaces need to be adjusted to balance the anti-torque generated by the single-rotor rotation before taking off, in order to avoid unnecessary deflection of the airframe which is harm to the steady flight of the UAV. The two wings on the $\mathrm{X}$ axis play the role of balancing the anti-torque in vertical flight, but in horizontal flight, they don't generate lift force, but they greatly increase weight of the body.

\subsection{Coaxial Dual-Rotor Tail-Sitter UAV Lift System}

Coaxial dual-rotor tail-sitter utilizes a propulsion system having a pair of brushless motor in coaxial configuration, which is shown as Figure 3. It balances the anti-torque of the rotors by the inverse rotating of the two rotors, to stabilize the flight attitude [4]. During the flight, the altitude of the UAV is controlled by decreasing or increasing the combined thrust of the two propellers. The yaw, pitch and roll motion is controlled via rudder and stabilizer installed below the two rotors, and the control scheme is shown in Table 2.

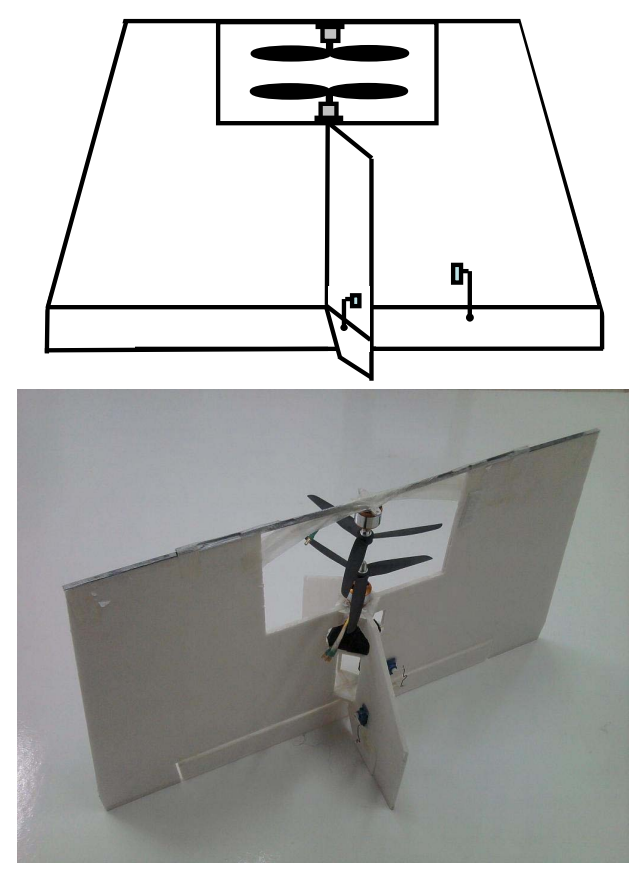

Figure 3. Coaxial dual-rotor tail-sitter UAV.
During the Vertical flight, the vehicle balances the anti-torque precisely by regulating the speed of the two motors to stabilize the flight attitude. Differential velocity of the two motors can control the yaw motion of the aircraft. Pitch motion of this configuration is controlled via the deflection of horizontal stabilizer. To control the roll motion, the aircraft moves the rudder. Converted from the vertical mode to the horizontal flight, the deflection of horizontal stabilizer is regulated until the UAV reaches the desired angle. During the horizontal mode, the aircraft is airborne and the forward motion is attained-air moving over the outer body wings and provides the lift for horizontal flight. In horizontal flight; the yaw motion is controlled by the rudder which provides the direction of the aircraft. When Pitch motion is changed to horizontal stabilizer motion, Roll motion is controlled by the differential velocity of the two motors and the vehicle thrust is regulated by the velocity of the propulsion system.

In coaxial dual-rotor lift system, the two motors are installed in the same axis direction precisely, and the speed difference between the two motors needs to be controlled within an allowable range. There are also higher requirements in the mechanical installation and control Alpha. However, in this lift system, if one motor failure, the aircraft can rely on the other motor to continue the flight through using the rudder to balance the anti-torque generated by the motor.

\subsection{Twins-Rotor Tail-Sitter UAV Lift System}

In the twin-rotor tail-sitter UAV Lift System, there are two brushless motors on the top of the airframe [5] as Shown in Figure 4. The motors rotate inversely to balance the anti-torque of each rotor. Decreasing or increasing the combined thrust of the two rotors could control the flight attitude. Two ailerons are installed under the two rotors. Yaw, pitch and roll motion of the aircraft are controlled through the deflection of ailerons and adjusting the speed of the two motors.

In the Vertical flight mode, the UAV balances the anti-torque precisely by regulating the speed of the two motors, to stabilize the flight attitude. Yaw motion is controlled through the Differential deflection of the two ailerons; while to control the pitch motion, two ailerons need to deflect in parallel. Differential velocity of the

Table 2. Control scheme of coaxial dual-rotor tail-sitter UAV.

\begin{tabular}{ccc}
\hline & VerticalMode & Horizontal Mode \\
\hline Yaw Motion & Differential velocity of the two motors & Rudde \\
Pitch Motion & Stabilizer & Differential velocity of the two motors \\
Roll Motion & Rudde & - \\
VTOL & Increase or decrease the combined thrust of the two motors & \\
\hline
\end{tabular}


two motors can control the roll motion of the aircraft. Decreasing or increasing the combined thrust of the two rotors could control the flight attitude. Converted from the vertical mode to the horizontal flight, the deflection of two ailerons is regulated until the UAV reaches the desired angle. In the horizontal mode, when forward motion of the aircraft is attained-air moving over the outer body wings provides the lift for horizontal flight. During horizontal flight, the yaw motion is controlled by the differential velocity of the two motors. Pitch motion is controlled by the parallel deflection of the two ailerons. Roll motion is controlled through differential deflection of the two ailerons. The vehicle thrust is regulated by the velocity of the propulsion system (see Table $\mathbf{3}$ ).

In the twin-rotor lift system, two motors need to be installed symmetrically in $\mathrm{Z}$ axis direction, and the speed of the two motors are required to controlled precisely, in order to stabilize the aircraft in flight. If one motor fails during the flight, the aircraft can't continue the flight relying on the other motor.

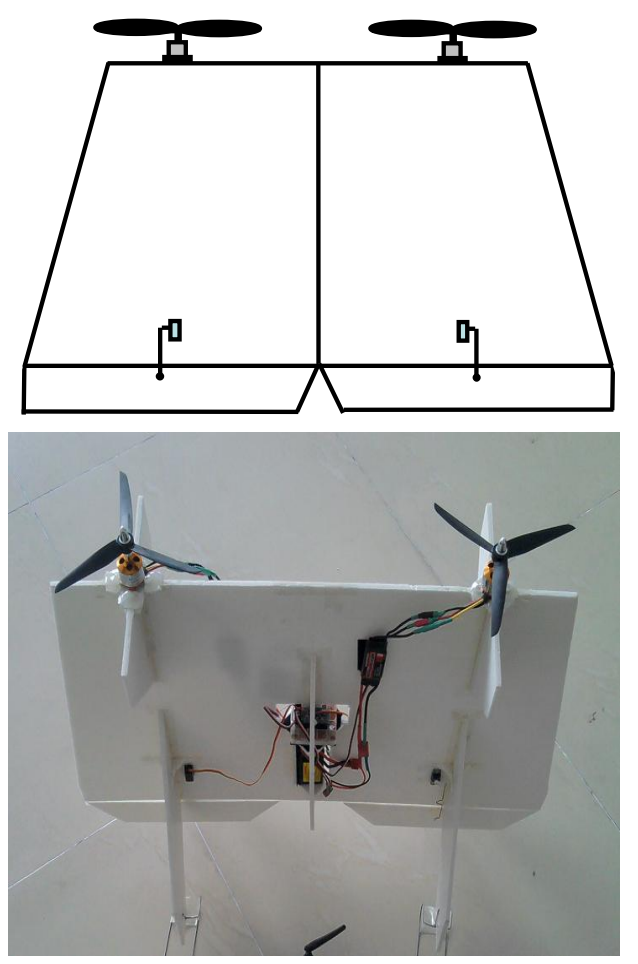

Figure 4. Twin-rotor tail-sitter UAV.

\section{Twin-Rotor Experimental Model of UAV}

According to the analysis and comparison of the three kinds of lift system above mentioned. The twin-rotor lift system can provide greater lift power compared with the single-rotor. Meanwhile, compared to the coaxial dualrotor, the twin-rotor is relatively simple in terms of mechanical installation .So we finally decide to adopt the twin-rotor lift system to manufacture an experimental UAV model. The well-made twin-rotor tail-sitter UAV is shown in Figure 5.

The airframe of the UAV is built of polystyrene foam sheet, and the vehicle is powered by propulsion systems which consists of two brushless motors juxtaposed on the airframe, which drive a pair of propellers of $90 \times 50$ in. The motor were driven by two 30A electronic speed controllers (ESC), and the power is provided by a Li-Poly battery. At the bottom of the airframe, two ailerons are installed under the downwash of the propellers. The deflection of the rudders is controlled by two servos, which is used to control the pitch and yaw motion of the aircraft in vertical flight. Parameters of this aircraft are shown in Table 4.

An inertial measurement unit (IMU) is placed in the UAV in order to measure the aircraft motion, which consists of three-axis digital gyroscope (L3G4200D), threeaxis digital accelerometer (ADXL345) and three-axis

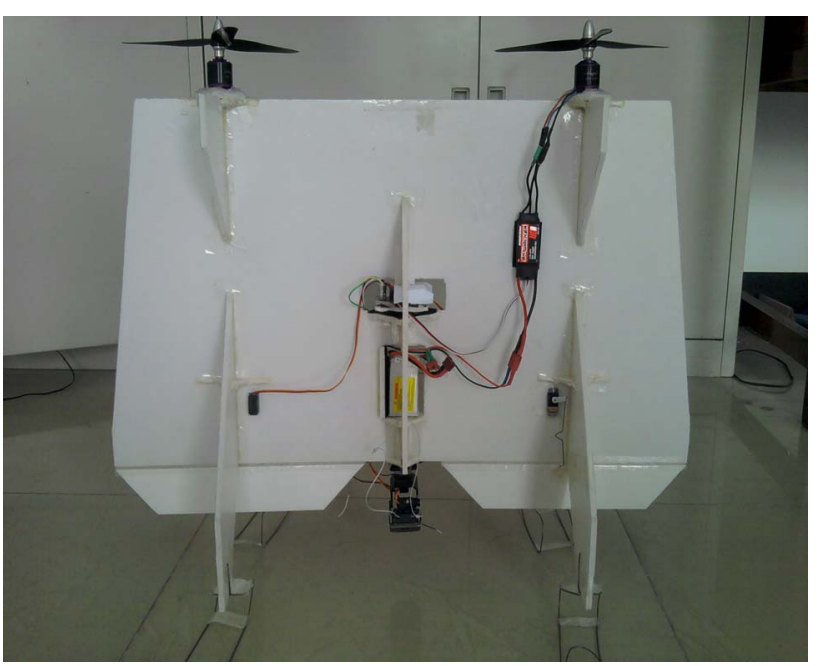

Figure 5. Well-made twin-rotor experimental UAV model.

Table 3. Control scheme of twin-rotor tail-sitter UAV.

\begin{tabular}{ccc}
\hline & Vertical Mode & Horizontal Mode \\
\hline $\begin{array}{l}\text { Yaw Motion } \\
\text { Pitch Motion }\end{array}$ & Differential deflection of the two ailerons & Differential velocity of the two motors \\
Roll Motion & Differential velocity of the two motors & Differential deflection of the two ailerons \\
VTOL & Increase or decrease the combined thrust of the two motors & -
\end{tabular}


Table 4. Parameters of the twin-rotor tail-sitter UAV.

\begin{tabular}{cc}
\hline Parameter & Value \\
\hline Airfoil shape & Plate \\
Wing chord & $0.5 \mathrm{~m}$ \\
Wing span & $0.75 \mathrm{~m}$ \\
Aspect ratio & 1.5 \\
Thickness & 0.007 \\
Wing area & $0.37 \mathrm{~m}^{2}$ \\
Mass vehicle & $0.73 \mathrm{~kg}$ \\
\hline
\end{tabular}

digital magnetometers (HMC5883L).

An inertial measurement unit (IMU) is placed in the UAV in order to measure the aircraft motion, which consists of three-axis digital gyroscope (L3G4200D), threeaxis digital accelerometer (ADXL345) and three-axis digital magnetometers (HMC5883L). The MCU (atmage328) receives and processes the data from the IMU in order to obtain a suitable control [6]. The MCU converts the flight information from the IMU into control signals for the motors and severs to control the flight speed of the UAV and the yaw, pitch and roll motion [7]. In Figure 6, (a) the aircraft is in the hover mode, (b) the aircraft is in horizontal flight. The aircraft finally can achieve the given height hover and high-speed horizontal flight under the suitable control law.

\section{Conclusions}

The experimental results show that the twin-rotor tailsitter UAV can provide large lift power and its flight attitude is flexible. During the vertical flight, the UAV can be controlled like a helicopter, and the transition maneuver from vertical to horizontal mode is quit easy control by the two ailerons deflecting in parallel. In the horizontal flight, the aircraft is controlled as an airborne. The twin-rotor lift system of the experimental UAV model is well designed and the mechanical installation of it is simple. The overall structure of the UAV is compact and flexible operated.

Although the flight experiment of the twin-rotor tailsitter UAV has been initially completed, but its automatic control law is far more complex than the common fixedwing aircraft and the accuracy requirements of attitude estimate is extraordinary higher. Therefore, future work should 1 be mainly focused on the research of control law. In addition, more sensors need to be added to the aircraft to achieve the navigation of autonomous flight. When incorporating GPS navigation and vision-based navigation [8], UAV will be controlled automatically taking off and landing in the urban environment, and complete the task of trajectory tracking and indoor vertical flight.

After the text edit has been completed, the paper is

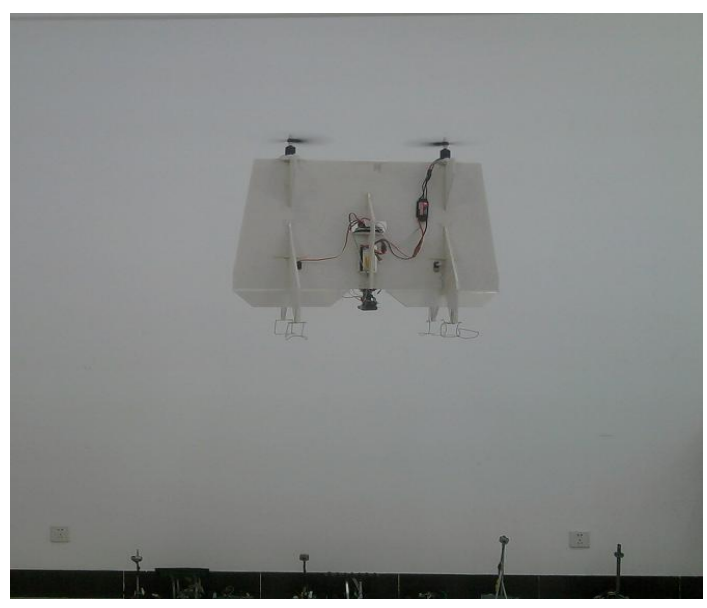

(a)

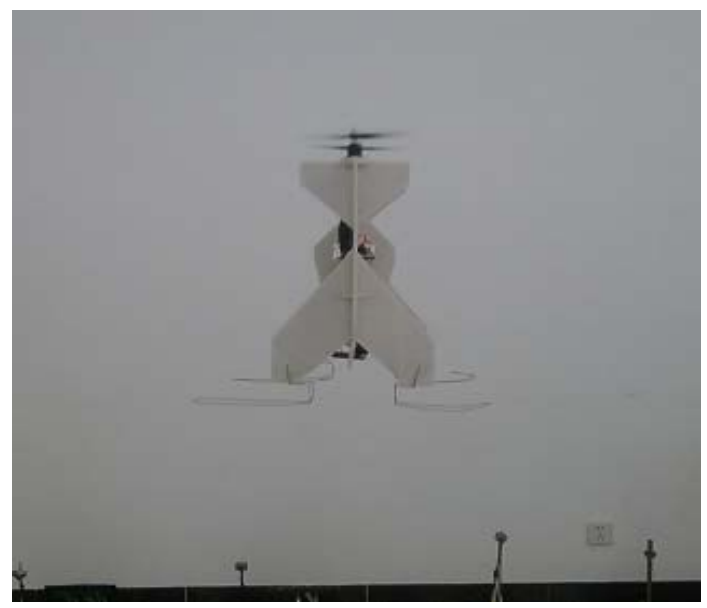

(b)

Figure 6. Twin-rotor experimental UAV model in Hover mode.

ready for the template. Duplicate the template file by using the save as command, and use the naming convention prescribed by your journal for the name of your paper. In this newly created file, highlight all of the contents and import your prepared text file. You are now ready to style your paper.

\section{REFERENCES}

[1] D. L. Kohlman, "Introduction to V/STOL Airplanes," Iowa State University Press, Oxford, 1981.

[2] H. Stone, "Aerodynamic Modelling of a Wing-in-Slipstream Tail-Sitter UAV,” 2002 Biennial International Powered Lift Conference and Exhibit, Williamsburg, 5-7 November 2002.

[3] M. A. GokhanInalhan, "Design Methodology of a Hybrid Propulsion Driven Electric Powered Miniature Tailsitter Unmanned Aerial Vehicle," Journal of Intelligent and Robotic Systems, Vol. 57, No. 1-4, 2010, pp. 505-529. doi:10.1007/s10846-009-9368-0

[4] J. Escareno, A. Sanchez, O. Garcia and R. Lozano, "Mo- 
deling and Global Control of the Longitudinal Dynamics of a Coaxial Convertible Mini-UAV in Hover Mode," Journal of Intelligent and Robotic Systems, Vol. 54, No. 1-3, 2009, pp. 261-273. doi:10.1007/s10846-008-9265-y

[5] J. Escareno, S. Salazar and R. Lozano, "Modelling and Control of a Convertible VTOL Aircraft," Proceedings of the 45th IEEE Conference on Decision \& Control, San Diego, 13-15 December 2006.

[6] J. Escareno, S. Salazar-Cruz and R. Lozano, "Embedded
Control of a Four-Rotor UAV,” Proceedings of the 2006 American Control Conference, Minneapolis, 14-16 June 2006.

[7] R. H. Stone, "Control Architecture for a Tail-Sitter Unmanned Air Vehicle," 5th Asian Control Conference, Melbourne, 20-23 July 2004.

[8] P. T. Millet, "Vision-Based Precision Landings of a Tailsitter UAV,” Master's Thesis, Brigham Young University, 2010. 